\title{
Andrology: Effects of nitric oxide on human spermatozoa: evidence that nitric oxide decreases sperm motility and induces sperm toxicity
}

\author{
Rosselli, Marinella ; Dubey, Raghvendra K ; Imthurn, Bruno ; Macas, Ervin ; Keller, Paul J
}

\begin{abstract}
Endogenous nitric oxide (NO) is an important functional mediator in several physiological systems, including the reproductive system. However, when generated in excessive amounts for long periods, mainly during immunological reactions, NO is cytotoxic and cytostatic for invading microbes, as well as for the cells generating it and the tissues present around it. Since infertility associated with urogenital tract infection in males and females is also accompanied by reduced sperm motility and viability, it is possible that reduced fertility in these patients is due to NO-induced sperm toxicity. We therefore evaluated the direct effects of NO, chemically derived from S-nitroso-N-acetylpenicillamine (SNAP, 0.012-0.6 $\mathrm{mM}$ ) and sodium nitroprusside (SNP, 0.25-2.5 mM), on the motility and viability of human spermatozoa. Furthermore, we tested whether inhibition of NO synthesis prevents sperm motility and viability by incubating washed total cells present in the semen (spermatozoa, round cells) with N-nitro-L-argininemethyl-ester (L-NAME), a NO synthesis inhibitor. Treatment of purified spermatozoa with SNAP or SNP decreased forward progressive sperm motility and straight line velocity, and also increased the percentage of immotile spermatozoa in a concentration-dependent manner. Furthermore, the percentage of immotile spermatozoa positively correlated with the percentage of dead spermatozoa. In contrast to freshly prepared SNAP, SNAP preincubated for $48 \mathrm{~h}$ had no effect on the motility and viability of the spermatozoa. Furthermore, as compared to untreated controls, a significantly higher percentage of forward progressive sperm motility as well as viability $(\mathrm{P}<0.05)$ was maintained in washed semen incubated with L-NAME (0.15 mM). Seminal plasma concentrations of nitrite-nitrate (stabile metabolites of NO/106 spermatozoa correlated positively $(\mathrm{P}<0.05)$ with the percentage of immotile spermatozoa. Our results suggest that NO can cause sperm toxicity as well as inhibit sperm motility. In conclusion, excessive NO synthesis in response to infection and inflammation could be an important factor contributing to functional change of the spermatozoa, leading to their dysfunction and to infertility
\end{abstract}

DOI: https://doi.org/10.1093/oxfordjournals.humrep.a136174

Posted at the Zurich Open Repository and Archive, University of Zurich

ZORA URL: https://doi.org/10.5167/uzh-154226

Journal Article

Published Version

Originally published at:

Rosselli, Marinella; Dubey, Raghvendra K; Imthurn, Bruno; Macas, Ervin; Keller, Paul J (1995). Andrology: Effects of nitric oxide on human spermatozoa: evidence that nitric oxide decreases sperm motility and induces sperm toxicity. Human Reproduction, 10(7):1786-1790.

DOI: https://doi.org/10.1093/oxfordjournals.humrep.a136174 


\section{Effects of nitric oxide on human spermatozoa: evidence that nitric oxide decreases sperm motility and induces sperm toxicity}

\author{
Marinella Rosselli ${ }^{1}$, Raghvendra K.Dubey ${ }^{2}$, \\ Bruno Imthurn, Ervin Macas and \\ Paul J.Keller
}

Department of Obstetrics and Gynaecology, Clinic of Endocrinology, University Hospital, 8091 Zurich, Switzerland and ${ }^{2}$ Center for Clinical Pharmacology, Medical School, University of Pittsburgh Medical Center, Pittsburgh, PA, USA

${ }^{1}$ To whom correspondence should be addressed

Endogenous nitric oxide (NO) is an important functional mediator in several physiological systems, including the reproductive system. However, when generated in excessive amounts for long periods, mainly during immunological reactions, NO is cytotoxic and cytostatic for invading microbes, as well as for the cells generating it and the tissues present around it. Since infertility associated with urogenital tract infection in males and females is also accompanied by reduced sperm motility and viability, it is possible that reduced fertility in these patients is due to NO-induced sperm toxicity. We therefore evaluated the direct effects of NO, chemically derived from $S$-nitroso- $N$ acetylpenicillamine (SNAP, 0.012-0.6 $\mathrm{mM}$ ) and sodium nitroprusside (SNP, 0.25-2.5 $\mathrm{mM}$ ), on the motility and viability of human spermatozoa. Furthermore, we tested whether inhibition of NO synthesis prevents sperm motility and viability by incubating washed total cells present in the semen (spermatozoa, round cells) with $N$-nitro-Larginine-methyl-ester (L-NAME), a NO synthesis inhibitor. Treatment of purified spermatozoa with SNAP or SNP decreased forward progressive sperm motility and straight line velocity, and also increased the percentage of immotile spermatozoa in a concentration-dependent manner. Furthermore, the percentage of immotile spermatozoa positively correlated with the percentage of dead spermatozoa. In contrast to freshly prepared SNAP, SNAP preincubated for $48 \mathrm{~h}$ had no effect on the motility and viability of the spermatozoa. Furthermore, as compared to untreated controls, a significantly higher percentage of forward progressive sperm motility as well as viability $(P<0.05)$ was maintained in washed semen incubated with L-NAME $(0.15$ mM). Seminal plasma concentrations of nitrite-nitrate (stabile metabolites of $\mathrm{NO}) / 10^{6}$ spermatozoa correlated positively $(P<0.05)$ with the percentage of immotile spermatozoa. Our results suggest that NO can cause sperm toxicity as well as inhibit sperm motility. In conclusion, excessive NO synthesis in response to infection and inflammation could be an important factor contributing to functional change of the spermatozoa, leading to their dysfunction and to infertility.

Key words: human sperm motility/nitric oxide

\section{Introduction}

Infertility during several forms of urogenital infection is associated with decreased sperm motility. This is preceded or accompanied by activation of lymphocytes and macrophages as well as the presence of an increased number of white blood cells in the semen (Haney et al., 1983; Anderson and Hill, 1988; Barratt et al., 1990), and depends on the extent of the infection and of the microorganism/spermatozoa ratio (Auroux et al., 1991). Several factors such as highly reactive oxygen free radicals, lymphokines and monokines generated by activated polymorphonuclear cells have been implicated in inducing infertility by causing sperm toxicity and reducing sperm motility (Aitken, 1989; Aitken et al., 1991; Plante et al., 1994). Cytokines, lipopolysaccharide and toxins generated during localized infection are known to activate white blood cells and smooth muscle cells to generate large amounts of nitric oxide (NO) continuously (Moncada et al., 1991; Nussler and Billiar, 1993; Morris and Billiar, 1994). Excessive NO in turn can have a cytotoxic and cytostatic effect on the invading microorganisms as well as the surrounding cells either directly, or in combination with free radicals (Moro et al., 1994; Morris and Billiar, 1994). It is therefore plausible that increased incidence of infertility in subjects with urogenital infection and inflammation is due to NO-induced sperm toxicity as well as reduced sperm motility.

NO is a labile and diffusible molecule which forms stable metabolites, nitrite/nitrate, that can be detected in biological fluids (Ochoa et al., 1991). NO is synthesized in a tightly regulated manner and is well known to be an important mediator in several physiological systems (Moncada et al., 1991; Nussler and Billiar, 1993; Moro et al., 1994; Morris and Billiar, 1994), including the reproductive system (Ellman et al., 1993; Yallampalli et al., 1993; Rosselli et al., 1994). $\mathrm{NO}$ is synthesized from a guanidine nitrogen atom of Larginine by the enzymatic action of the NO synthase system, which can be inhibited by competitive $L$-arginine analogues like $N$-nitro-L-arginine-methyl-ester (L-NAME) (Moncada et al., 1991). NO synthase exists in three distinct isoforms, two of which are continuously present and termed constitutive NO synthase. The third isoform is inducible and present in macrophages, monocytes and smooth muscle cells; its activation by certain cytokines or microbial products results in a sustained production of NO (Nussler and Billiar, 1993).

The primary mechanism of NO-induced cytostaticity and cytotoxicity includes direct inhibition of mitochondrial respiration and DNA synthesis (Hibbs et al., 1990). Furthermore, NO toxicity is also mediated indirectly through its interaction with other molecules, mainly superoxide anions $\left(\mathrm{O}_{2}^{-}\right)$and formation of peroxynitrite anion $\left(\mathrm{ONOO}^{-}\right)$, which when protonated, decomposes to form ${ }^{\circ} \mathrm{OH}$ and $\mathrm{NO}_{2}$, both of which are 
tissue-damaging agents (Beckman et al., 1990; Ischiropoulos et al., 1992; Moro et al., 1994). Compared to NO and superoxide free radical alone, peroxynitrite (their reactive product) is several times more toxic (Moro et al., 1994). Based on the above facts, we postulate that excessive NO generation and/or concentration causes cytotoxicity to spermatozoa and decreases sperm motility. Furthermore, in infertility associated with microbial infection, excessive production of NO may mainly contribute to the sperm dysfunction.

In the present study, we evaluated the direct effects of NO, chemically generated from SNAP ( $S$-nitroso- $N$-acetylpenicillamine) or SNP (sodium nitroprusside), on the motility and viability of purified human spermatozoa. Additionally, using a non-purified sperm preparation (containing round cells) the direct evidence for NO-mediated sperm toxicity was evaluated by testing the effect of NO synthase inhibition by L-NAME on sperm motility and viability. Further, to confirm whether changes in endogenous NO concentrations are also accompanied by parallel changes in sperm functionality, we directly measured the concentrations of nitrite-nitrate in the seminal plasma obtained from different patients and we correlated its concentration with sperm motility.

\section{Materials and methods}

\section{Semen collection}

Sperm samples were obtained from healthy subjects who underwent a semen analysis for couple infertility. Specimens depicting normozoospermia (rapid forward + non-rapid forward motile spermatozoa $>50 \%$ ) in accordance with the World Health Organization guidelines (WHO laboratory manual, 1987) or light asthenozoospermia (rapid forward + non-rapid forward motile spermatozoa $>38 \%$ ) were used for the study.

\section{Effect of SNP and SNAP on sperm motility}

Semen samples $(n=5)$ for each set of experiments were layered on a pre-established two-step 90, 47\% Percoll (Pharmacia, Uppsula, Sweden) gradient buffered with Ham's F10 medium (Sigma, Basel, Switzerland). Gradients were centrifuged for $20 \mathrm{~min}$ at $300 \mathrm{~g}$. Spermatozoa present in the pellet were resuspended at $30 \pm 10 \times 10^{6}$ spermatozoa/ml in incubation medium [Ham's F10 with $1 \%$ fetal calf serum (FCS; Gibco, Basel, Switzerland), $25 \mathrm{mM} \mathrm{L-(+)-sodium} \mathrm{lactate}$ (Sigma), $0.33 \mathrm{mM}$ sodium pyruvate (Sigma)]. Aliquot $(200 \mu \mathrm{l})$ of spermatozoa suspension treated with or without SNP $(0.25,1.25,2.5$ $\mathrm{mM}$; Sigma) or SNAP $(0.012,0.12,0.6 \mathrm{mM}$; Alexis Corporation, Läufelfingen, Switzerland) or $0.12 \mathrm{mM}$ SNAP preincubated at room temperature for $48 \mathrm{~h}$ were kept at room temperature under sterile conditions. Motility was assessed with a computer-assisted sperm motility analyser (Motility Analyzer; Mika Medical GmbH, Germany) after overnight incubation.

Percentages of rapid forward, non-rapid forward (local) and immotile spermatozoa were classified according to WHO (1987) guidelines. Straight line velocity (VSL) was obtained from all the motile spermatozoa.

A vitality test was performed using the eosin vital live-dead staining method (Berthelsen, 1981). All data represent the mean \pm $\mathrm{SE}$ of the means of five different experiments.

\section{Effect of L-NAME on sperm motility}

Semen specimens $(n=7)$ were diluted 1:4 with incubation medium [Ham's F10 with 1\% FCS, $25 \mathrm{mM} \mathrm{L}$-(+)-sodium lactate, $0.33 \mathrm{mM}$ sodium pyruvate] and centrifuged for $10 \mathrm{~min}$ at $300 \mathrm{~g}$. Pellets containing spermatozoa and other cells present in the ejaculate were resuspended in $5 \mathrm{ml}$ of incubation medium and recentrifuged for 10 $\mathrm{min}$ at $300 \mathrm{~g}$. The final pellets were resuspended in incubation medium at $30 \pm 10 \times 10^{6}$ spermatozoa/ml. Aliquots of sperm suspension ( 200 $\mu \mathrm{l})$ were incubated under sterile conditions, at room temperature, in the absence (control) or presence of $0.150 \mathrm{mM}$ L-NAME (Calbiochem, Läufelingen, Switzerland). Motility was assayed with a computerassisted sperm motility analyser after $4 \mathrm{~h}$ incubation.

\section{Nitrite-nitrate analysis}

Seminal plasma were obtained from different subjects $(n=13)$ after centrifugation of the semen at $300 \mathrm{~g}$ for $10 \mathrm{~min}$. Before nitrite-nitrate analysis, seminal plasma was recentrifuged at $8000 \mathrm{~g}$ for $15 \mathrm{~min}$. Nitrite-nitrate concentrations were then measured using the Greiss reagent as described before (Green et al., 1982; Ochoa et al. 1991). Briefly, seminal plasma aliquots $(250 \mu \mathrm{l})$ were diluted with ultrapure water ( $500 \mu \mathrm{l}$; Seromed Biochrom, Berlin, Germany) and incubated at room temperature with $250 \mu \mathrm{l}$ of substrate buffer (imidazole 0.1 $\mathrm{mol} / \mathrm{l}$, NADPH $210 \mu \mathrm{mol} / \mathrm{l}$, flavine adenine dinucleotide $3.8 \mu \mathrm{mol} / \mathrm{l}$; $\mathrm{pH} 7.6$ ) in the presence of nitrate-reductase (Aspergillus niger, 70 IU/l; Boehringer Mannheim, Rotkreuz, Switzerland) for $45 \mathrm{~min}$, to convert nitrate to nitrite. Total nitrite was then analysed by reacting the samples with Greiss reagent ( $1 \%$ sulfanilamide, $0.1 \%$ naphthaleneethylene diamine dihydrochloride in $5 \% \mathrm{H}_{3} \mathrm{PO}_{4}$, Spectroquant; Merck, Darmstadt, Germany). Reacted samples were treated with $500 \mu \mathrm{l}$ of trichloroacetic acid (20\%), centrifuged for $15 \mathrm{~min}$ at $8000 \mathrm{~g}$ and the absorbence of the supernatant was measured spectrophotometrically at $520 \mathrm{~nm}$. Amounts of nitrite-nitrate in seminal plasma were estimated from a standard curve of $\mathrm{NaNO}_{2}$ obtained by enzymatic conversion of $\mathrm{NaNO}_{3}(0-32 \mathrm{nmol} / \mathrm{ml}$; Merck $)$. The measured concentration was normalized to the number of $10^{6} \mathrm{spermatozoa} / \mathrm{ml}$.

\section{Statistics}

Comparison between the untreated and treated samples was made using Student's $t$-test and a $P$ value of $<0.05$ was considered significant. Linear regression analysis and Spearman's rank correlation coefficient were used to determine the correlation between the concentrations of nitrite-nitrate $/ 10^{6}$ spermatozoa and the percentage of immotile spermatozoa; $P<0.05$ was considered significant.

\section{Results}

\section{Effects of chemically derived NO on sperm motility and viability}

SNAP releases large amounts of NO spontaneously in aqueous media, whereas SNP releases NO either spontaneously or by intracellular catalysed reaction (Ignarro et al., 1981; Marks et al., 1991; Kowaluk et al., 1992), although the exact mechanism still remains unclear. The effects of NO-generating compounds on sperm motility are shown in Figure $1 \mathrm{~A}$ and $\mathrm{B}$. Treatment of spermatozoa with SNAP or SNP significantly decreased the percentage of spermatozoa expressing rapid forward motility as well as an increase in the percentage of immotile spermatozoa in a concentration-dependent manner. The threshold concentration at which these NO donors had a significant effect on sperm motility was $0.12 \mathrm{mM}$ for SNAP and $0.25 \mathrm{mM}$ for SNP.

After vital staining we could observe that the profile of decreasing spermatozoa vitality in response to SNAP or SNP 

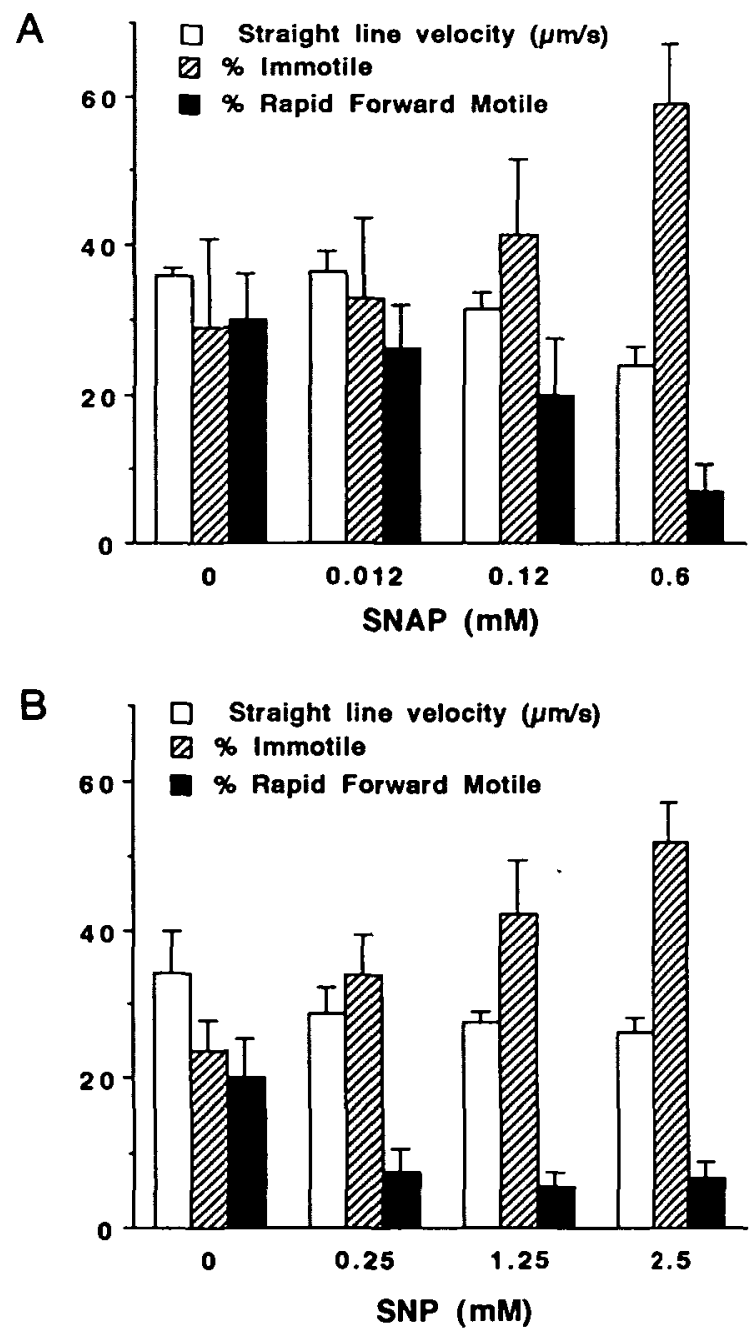

Figure 1. The effect of (A) $S$-nitroso- $N$-acetylpenicillamine (SNAP) and (B) sodium nitroprusside (SNP) on sperm motility. Treatment of human spermatozoa with SNAP or SNP significantly decreases rapid forward sperm motility (progression) in a concentrationdependent manner and also increases the number of immotile spermatozoa in a concentration-dependent manner. Furthermore, treatment of human spermotozoa with SNAP or SNP decreases straight line velocity (VSL) in a concentration-dependent manner.

was similar and parallel to the increase in the number of immotile spermatozoa.

Since the motility of spermatozoa has been characterized based on their velocity and pattern of movement, the effects of these compounds were also evaluated on the straight line velocity (VSL) of the total motile spermatozoa. Treatment of spermatozoa with SNAP or SNP reduced the VSL of the spermatozoa in a concentration dependent manner (Figure lA and B). Of the two chemically dissimilar NO-generating compounds, SNAP, which generates more NO than SNP, was more potent in decreasing the VSL of the spermatozoa population.

To confirm that the inhibitory effect was due to NO and not due to the compounds themselves, the effect of freshly prepared SNAP on spermatozoa motility was compared with that of SNAP that had been preincubated for $48 \mathrm{~h}$. No significant difference was observed between the untreated spermatozoa

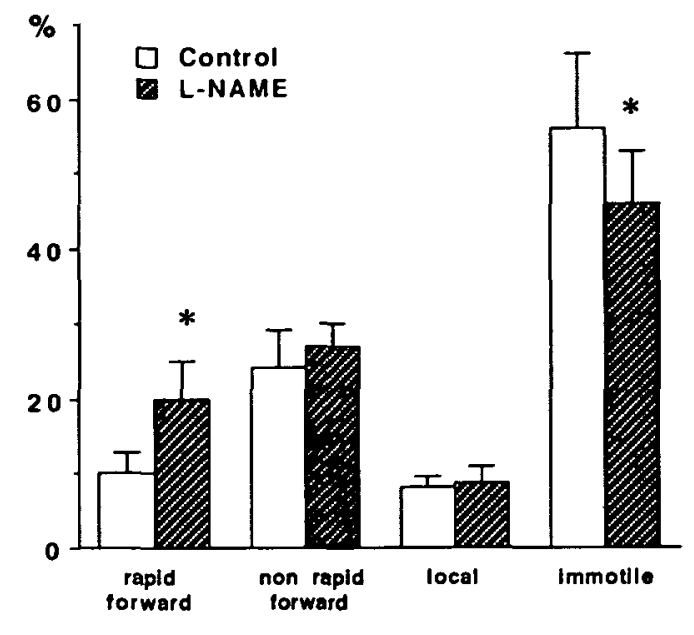

Figure 2. The effect of $N$-nitro-L-arginine-methyl-ester (L-NAME; $0.150 \mathrm{mM}$ ) on human sperm rapid forward motility, non-rapid forward motility, local motility and immotility. ${ }^{*}=$ significantly different $(P<0.05)$. Control represents the motility of the washed semen but in the absence of L-NAME.

and the spermatozoa treated with $0.12 \mathrm{mM}$ SNAP preincubated for $48 \mathrm{~h}$.

\section{Effect of a NO synthesis inhibitor (L-NAME) on spermatozoa motility and vitality}

To study the effect of L-NAME on spermatozoa motility and vitality, we used a sperm preparation from which contaminating round cells (polymorphonuclear cells and germ cells) and bacteria were not eliminated. The effect of L-NAME is shown in Figure 2. When compared with untreated sperm samples, the rapid forward motility of the spermatozoa was significantly $(P<0.05)$ maintained in spermatozoa incubated for $4 \mathrm{~h}$ with $0.150 \mathrm{mM}$ L-NAME. No significant changes were observed between the non-rapid forward motile spermatozoa as well as the local motile spermatozoa. Furthermore, the immotile spermatozoa population significantly decreased in the presence of L-NAME $(P<0.05)$.

\section{Nitrite-nitrate concentrations in seminal plasma}

The concentrations of nitrite-nitrate analysed in seminal plasma ( $n=13$ ) of different subjects ranged from 16 to $50 \mathrm{nmol} / \mathrm{ml}$. A significant positive correlation $(r=0.740, P<0.01$; Spearman rank coefficient $=0.67, P<0.05$ ) was observed between seminal plasma concentrations of nitrite-nitrate ( $\mathrm{nmol} /$ $10^{6}$ spermatozoa) and the percentage of immotile spermatozoa in the different semen samples (Figure 3).

\section{Discussion}

In the present study we provide the first evidence that NO can induce toxicity as well as decrease forward progressive motility in human spermatozoa. NO is known to be an important mediator in several physiological systems (Moncada et al., 1991) and has beneficial biological effects when generated within physiological limits. When generated in large quantities for long periods, mainly during immunological reactions, NO is cytostatic and cytotoxic for invading microorganisms and 


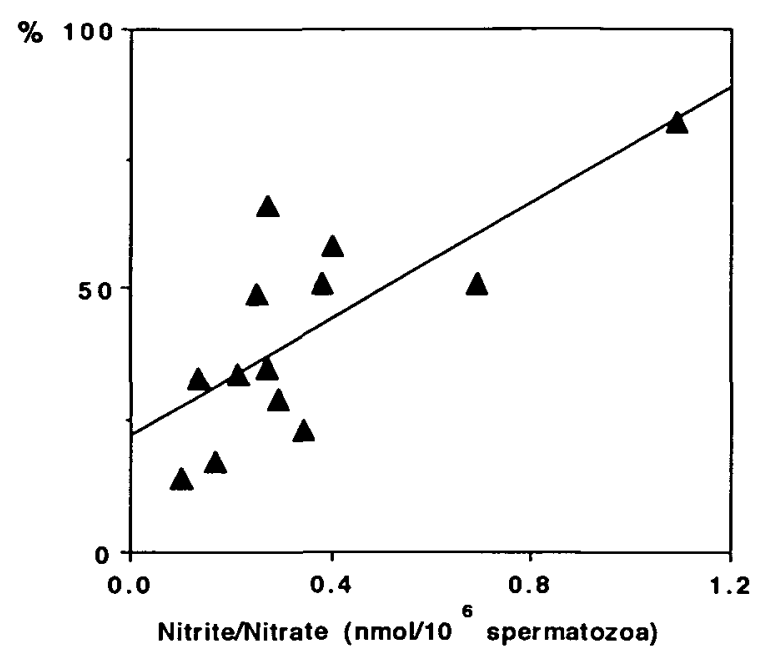

Figure 3. The correlation between the nitrite-nitrate concentrations (nmol/ $10^{6}$ spermatozoa) in seminal plasma and the percentage of immotile spermatozoa $(r=0.74, P<0.01$; Spearman rank coefficient $=0.677, P<0.05$ )

might also damage the tissues that generate it (Morris and Billiar, 1994; Nussler and Billiar, 1993). Together with the observation that clinical studies have shown that in humans, female and male, infection of the reproductive tract is associated with increased incidence of infertility (Haney et al., 1983; Barratt et al., 1990; Glezerman and Bartoov, 1993), we postulate that pathologically increased NO synthesis might, at least in part, be responsible for causing infertility in infected patients, possibly by causing spermatozoa death and by decreasing sperm motility.

In the present study this hypothesis was tested by studying the effects of NO generated from SNAP and SNP on sperm motility and viability. Furthermore, the possibility that inhibition of NO synthesis by L-NAME improves sperm motility and maintains sperm viability was also investigated.

Our observation that both SNP and SNAP decreased sperm motility in a concentration-dependent manner suggests that increased NO concentrations are toxic and decrease sperm viability. Indeed, the observed increases in the percentage of immotile spermatozoa were also accompanied by a parallel change in the percentage of dead spermatozoa. Compared to SNP, SNAP was more potent in decreasing sperm velocity. Differences in toxic potency between these compounds may be largely attributable to differences in the mechanisms by which they generate NO. SNAP releases NO spontaneously in aqueous media (Ignarro et al., 1991; Marks et al., 1991; Kowaluk et al., 1992; Dubey, 1994), whereas the mechanism by which SNP generates NO still remains undefined. Previously it was assumed that SNP releases NO by spontaneous chemical degradation. However, more recently it has been shown that generation of NO from SNP requires an intracellular or membrane catalysed metabolic reaction (Ignarro et al., 1981; Marks et al., 1991; Kowulak et al., 1992).

The observation that SNP and SNAP decrease sperm motility and viability could only suggest the involvement of NO. However, the possibility that the toxic effects were caused by the chemicals themselves could not be ruled out from these experiments. Hence, the role of NO in mediating the toxic effect was confirmed by comparing the effect of freshly prepared SNAP on sperm motility with that of SNAP that had been preincubated for $48 \mathrm{~h}$. Our observation that sperm motility decreases in response to freshly prepared SNAP but not to preincubated SNAP further substantiates the evidence that the toxic effects were not due to the compound itself, but rather to a consequence of NO generation.

Since leukocytes and macrophages as well as bacteria are present in human seminal plasma (Anderson and Hill, 1988) and macrophages are capable of generating large amounts of NO during an immune reaction (Nussler and Billiar, 1993), we evaluated whether inhibition of NO synthesis by L-NAME improves sperm motility. This set of experiments was conducted without removing the contaminating cells, i.e. macrophages, leukocytes and bacteria. Our observation that motility and viability were significantly maintained in samples treated with L-NAME as compared to untreated spermatozoa strongly suggests that increased synthesis of NO causes sperm damage and may be also one of the factors contributing to infertility.

The notion that NO can induce sperm toxicity was further confirmed by our observation that in the native semen collected from different subjects the concentrations of nitrite-nitrate (stable metabolites of NO) positively correlated with the percentage of immotile spermatozoa. Furthermore, a correlation between increased nitrite-nitrate concentrations in seminal plasma and a decreased percentage of rapid progressive motile spermatozoa was also observed (data not shown). Hence, these results suggest that the increases in NO synthesis in vivo can also affect sperm viability.

It is well established that in several pathological situations related to systemic as well as non-systemic infection there is a continuous release of excessive amounts of NO (Morris and Billiar, 1994). The increase in NO synthesis is mediated via inflammatory factors such as endotoxins and cytokines capable of inducing NO synthesis activity (Moncada et al., 1991). NO synthase activity is also localized in the oviduct (Rosselli et al., 1994), the conduit for the gametes to reach the oocyte and the environment where fertilization occurs. Apart from the effort of the spermatozoa, the rhythmic contraction of the oviduct also promotes and contributes to the process of sperm movement and fertilization. Hence, pathological conditions such as infection of the oviduct or endometriosis might also result in generation of excessive amounts of $\mathrm{NO}$, which not only would impair the normal contractility of the oviduct, but also damage the movement of the spermatozoa, causing sperm toxicity and consequently resulting in infertility.

In contrast to our observations, Hellstrom et al. (1994) recently reported that NO generated from SNP improves sperm motility and viability and suggested that these protective effects of NO are due to its capability to react with superoxide free radicals, which are known to inflict cell toxicity (Hill et al., 1987; Plante et al., 1994), and hence NO would neutralize the cytotoxic effects of the free radicals on spermatozoa. Although attractive, their explanation is flawed by the documented fact that within the biological system NO effectively reacts with superoxide anion $\left(\mathrm{O}^{2-}\right)$, forming a highly toxic peroxynitrite anion $\left(\mathrm{ONOO}^{-}\right)$(Beckman et al., 1990; Ischiropoulos et al., 
1992; Moro et al., 1994). This powerful oxidant, when protonated, decomposes rapidly, resulting in the formation of $\mathrm{OH}^{*}$ and $\mathrm{NO}_{2}$ both of which are tissue damaging agents (Beckman et al., 1990). Another explanation to the difference between this study and that reported by Hellstrom et al. (Hellstrom et al., 1994) could be the differences in concentration of SNP used and the duration of the incubation.

The mechanism by which NO induces its cytotoxic effect on human spermatozoa can only be speculated. Since NO is known to activate guanylate cyclase activity (Moncada et al., 1991) and increase cGMP formation, it is possible that the increased cGMP synthesis could be inducing these effects. Indeed, it has been demonstrated that non-physiological concentrations of cGMP decrease human sperm motility (Chan et al., 1990). Furthermore, it is conceivable that increased formation of $\mathrm{NO}$ and $\mathrm{O}_{2}^{-}$under pathological conditions consequently leads to the formation of $\mathrm{ONOO}^{-}$, which could directly interact with sperm membranes leading to sperm dysfunction and toxicity.

In conclusion, we provide evidence that NO can decrease sperm motility as well as viability, an effect that can be abrogated by inhibiting NO synthesis by L-NAME. Based on our finding it could be speculated that NO generated in nonphysiological/excessive amounts by macrophages in response to invading organisms could be one of the factors which induce dysfunction in spermatozoa and consequently increase infertility. The exact mechanism of such a process needs to be further investigated.

\section{Acknowledgement}

The excellent technical assistance of Ms Marika Borsos and Mrs Catherine Zurbrügg of the Endocrinology Laboratory, University Hospital, Zurich is gratefully acknowledged.

\section{References}

Aitken, R.J. (1989) The role of free oxygen radicals and sperm function. Int. J. Androl., 12, 95-97.

Aitken, J.R., Irvine, D.S. and Wu, F.C. (1991) Prospective analysis of spermoocyte fusion and reactive oxygen species generation as criteria for the diagnosis of infertility. Am. J. Obstet. Gynecol., 164, 542-551.

Anderson, D.J. and Hill, J.A. (1988) Cell mediated immunity and infertility. Am. J. Reprod. Immunol. Microbiol, 17, 22-30.

Auroux, M.R., Jacques, L., Mathreu, D. and Auer, J. (1991) Is the sperm bacterial ratio a determining factor in impairment of sperm motility: an in vitro study in man with Escherichia coli. Int. J. Androl., 14, 264-270.

Bartatt, C.L.R., Bolton, A.E. and Cooke, I.D. (1990) Functional significance of white blood cells in the male and the female reproductive tract. Hum. Reprod., 5, 639-648.

Beckman, J.S., Beckman, T.W., Chen, J., Marshall, P.A. and Freeman, B.A. (1990) Apparent hydroxyl radical production by peroxynitrite: implications for endothelial injury from nitric oxide and superoxide. Proc. Natl. Acad. Sci. USA, 87, 1620-1624.

Berthelsen, J.G. (1981) Vital staining of spermatozoa performed by the patient. Fertil. Sieril., 35, 86-87.

Chan. Y.M., Chan, S.Y.. Tucker. M.J.. Wong. C.J., Leung, C.K. and Leong, M.K. (1990) Effects of dibutyryl cyclic guanosine monophosphate on human spermatozoa motility and penetration of zona-free hamster oocyte. Hum. Reprod., 5, 304-308.

Dubey, R.K. (1994) Vasodilator-derived nitric oxide inhibits fetal calf serumand angiotensin-II induced growth of renal arteriolar smooth muscle cells. J. Pharmacol. Exp. Ther, 269, 402-408.

Ellman, C.. Corbett. J.A.. Misko, T.P.. McDaniel. M. and Beckerman, K.P.
(1993) Nıtric oxide mediates interleukin-1-induced cellular cytotoxicity in the rat ovary. A potential role for nitric oxide in the ovulatory process. J. Clin. Invest., 92, 3053-3056.

Glezerman, M. and Bartoov, B. (1993) Semen analysis. In Insler, V. and Lunenfeld, B. (eds), Infertility, Male and Female, 2nd edn. Churchill Livingstone, Edinburgh, pp. 285-315.

Green, L.C., Wagner, D.A., Glowski, J., Skipper, P.L. Wishnok, J.S. and Tannenbaum, S.R. (1982) Analysis of nitrite and $\left({ }^{15} \mathrm{~N}\right)$ nitrate in biological fluids. Anal. Biochem., 126, 131-138.

Haney, A.F., Misukonis, M.A. and Weinberg, J.B. (1983) Macrophages and infertility: oviductal macrophages as potential mediators of infertility. Fertil. Steril., 39, 310-315.

Hellstrom, W.J.G., Bell, M., Wang, R. and Sikka, S.C. (1994) Effect of sodium nitroprusside on sperm motility, viability, and lipid peroxidation. Fertil. Steril., 61, 1117-1122.

Hibbs, J.B., Jr, Taintor, R.R., Vavrin, Z., Granger, D.L., Drapier, J.C., Amber, I.J. and Lancaster, J.R., Jr (1990) Synthesis of nitric oxide from a terminal guanidine nitrogen atom of L-arginine: a molecular mechanism regulating cellular proliferation that targets intracellular iron. In Moncada, S. and Higgs, E.A. (eds), Nitric Oxide from L-Arginine: A Bioregulator System. Elsevier, Amsterdam, pp. 189-223.

Hill, J.A., Haimovic, F., Politch, J.A. and Anderson, D.J. (1987) Effects of soluble products of activated lymphocytes and macrophages (lymphokines and monokines) on human sperm motion parameters. Fertil. Steril., 47, $460-465$.

Ignarro, L.J., Lippton, H., Edwards, J.C., Baricos, W.H., Hyman, A.L., Kadowitz, P.J. and Gruetter, C.A. (1981) Mechanism of vascular smooth muscle relaxation by organic nitrates, nitrites, nitroprusside and nitric oxide: evidence for involvement of S-nitrosothiols as active intermediates. J. Pharmacol. Exp. Ther, 218, 739-749.

Ischiropoulos, H., Zhu, L. and Beckman, J.S. (1992) Peroxynitrite formation from macrophage-derived nitric oxide. Arch. Biochem. Biophys., 298, 446-451.

Kowulak, E.A., Seth, P. and Fung, H.L. (1992) Metabolic activation of sodium nitroprusside to nitric oxıde in vascular smooth muscle. J. Pharmacol. Exp. Ther, 262, 916-922.

Marks, G.S., McLaughlin, B.E., Brown, L.B., Beaton, D.E., Nakatsu, K. and Brien, J.F. (1991) Interaction of glycerol trinitrate and sodium nitroprusside with bovine pulmonary vein homogenate and 10000 $\mathrm{x} g$ supernatant: biotransformation and nitric oxide formation. Can. J. Physiol. Pharamcol., 69, 889-892.

Moncada, S., Palmer, R.M. and Higgs, E.A. (1991) Nitric oxide: physiology pathophysiology and pharmacology. Pharmacol. Rev., 43, 109-142.

Moro, M.A., Darley-Usmar, V., Goodwin, D.A., Read, N.G., Zamora-Pino, R., Feelisch, M., Radomski, M.W. and Moncada, S. (1994) Paradoxical fate and biological action of peroxinitrite on human platelet. Proc. Natl. Acad. Sci. USA, 91, 6702-6706.

Morris, S.M. and Billiar, T.R. (1994) New insights into the regulation of inducible nitric oxide synthesis. Am. J. Physiol., 266, E829-E839.

Nussler, A.K. and Billiar, T.R. (1993) Inflammation, immunoregulation, and inducible nitric oxide synthase. J. Leukocyte Bıol., 54,171-178.

Ochoa, J.B., Udekwu, O.A., Billiar, T.R., Curran, R.D., Cerra, F.B., Simmsons, R.L. and Peitzman. A.B. (1991) Nitrogen oxide levels in patients after trauma and during sepsis. Ann. Surg., 214, 612-626.

Plante, M., de Lamirande, E. and Gagnon, C. (1994) Reacuve oxygen species released by activated neutrophils, but not by deficient spermatozoa are sufficient to affect normal sperm motility. Fertil. Steril., 62, 387-393.

Rossellı, M., Imthum, B., Macas, E., Keller, P.J. and Dubey, R.K. (1994) Endogenous nitric oxide modulate endothelin-l induced contraction of bovine oviduct. Biochem. Biophys. Res. Commun., 201, 143-146.

World Health Organization (1987) WHO collection and examination of human semen. In Laboratory Manual for the Examination of Human Semen and Semen-Cervical Mucus Interaction, 2nd edn. University of Cambridge Press. Cambridge, pp. 1-16.

Yallampalli, C., Izumi, H., Byam-Smith, M. and Garfield, R.E. (1993) An Larginine-nitric-oxide-cyclic guanosine monophosphate system exists in the uterus and inhibits contractility during pregnancy. Am. J. Obstet. Gynecol.. $170,175-185$.

Received on January 13, 1995; accepted on April 7, 1995 